Review article

Paediatrics Today 2016;12(1):16-29

DOI $10.5457 / \mathrm{p} 2005-114.132$

\title{
THE NEONATAL CHEST
}

\author{
Veronica DONOGHUE* \\ The National Maternity Hospital \\ Dublin 2, Ireland \\ "Corresponding author: \\ vdonoghue@gmail.com \\ Tel.: + 3531637352
}

Received: January 10, 2016

Accepted: January 25, 2016

Key words: Hyaline membrane disease

- Meconium aspiration syndrome -

Congenital pneumonia - Congenital

pulmonary airway malformation -

Congenital diaphragmatic hernia.

\begin{abstract}
The aim of this paper is to describe the most common medical and surgical chest conditions encountered in the premature and term infant, by neonatologists and neonatal surgeons working in a newborn intensive care unit. The paper also reflects the imaging findings as a result of the many advances in the medical treatments used in the treatment of these chest conditions. It also addresses the issues relating to the very premature infants less than 26 weeks gestation. Advances in antenatal imaging lead to antenatal diagnoses in many of the surgical conditions encountered, which allows better postnatal planning and understanding of many of these conditions. Conclusion - Despite the many advances in imaging the Chest Radiograph still remains the primary source of information in the various chest abnormalities in these very sick infants.
\end{abstract}

\section{Introduction}

Over the past 30 years there have been many advances in the treatment of critically ill neonates. This has resulted in the survival and decreased morbidity among the term and preterm infants and in recent years this has extended to include infants of lower gestational ages. Many of these advances are related to the better management of neonatal lung conditions such as respiratory distress syndrome in the premature infant and meconium aspiration syndrome and persistent pulmonary hypertension of the newborn (PPHN) in the term infant together with the better understanding of the various congenital chest abnormalities. There have been key advances in mechanical ventilation, such as high frequency ventilation and extracorporeal membrane oxygenation (ECMO) and new drug therapies such as artificial surfactant and nitric oxide.
Advances in ultrasound equipment and the addition of fetal magnetic resonance imaging (MRI) have led to an improvement in the diagnosis of congenital lung lesions and have also added to our understanding of their evolution.

\section{Normal lung development}

The normal lung development is well described by Agrons et al. (1). During the embryonic phase of gestation (from 26 days to 6 weeks) the lung bud develops from the primitive foregut and divides to form the early tracheobronchial tree. During the pseudoglandular phase ( 6 to 16 weeks) there is airway development to the level of the terminal bronchioles, with a deficient number of alveolar saccules. Multiple alveolar ducts develop from the respiratory bronchioles during the cannicular or acinar phase (16 to 28 weeks). These ducts are lined by Type II alveolar cells 
which can produce surfactant, and which differentiate into thin Type I alveolar lining cells. At the end of this phase primitive alveoli form. Progressive thinning of the pulmonary interstitium allows gas exchange with approximation of the proliferating capillaries and the Type I cells. During the saccular phase (28 to 34 weeks) there is an increase in the number of terminal sacs, further thinning of the interstitium, continuing proliferation of the capillary bed and early development of the true alveoli. The alveolar phase extends from approximately 36 week gestation until 18 month of age with most alveoli formed at 5-6 months of age.

\section{Medical conditions affecting the chest in the newborn}

The common medical conditions which present in the newborn include Hyaline Membrane Disease (HMD) in the premature infant, and Transient Tachypnoea of the Newborn (TTN), Meconium Aspiration Syndrome (MAS), Congenital Pneumonia and Spontaneous Pneumothoraces in the term infant. While they occasionally present in the newborn period, other congenital surfactant deficient disorders are extremely rare

\section{Hyaline membrane disease (HMD) or idiopathic respiratory distress syndrome}

Idiopathic respiratory distress syndrome (IRDS) or Hyaline membrane disease (HMD) mainly affects the premature infant less than 36 weeks gestational age. The basic problem in HMD is a deficiency of the lipoprotein pulmonary surfactant in association with structural immaturity of the lungs. The lipoproteins are produced in the Type II pneumocytes, are concentrated in the cell lamellar bodies and then transported to the cell surface and expressed onto the alveolar luminal surface. These lipoproteins then combine with surface surfactant proteins $(\mathrm{A}, \mathrm{B}, \mathrm{C}, \mathrm{D})$ which are also produced by the Type II pneumocytes to form tubular myelin. This is the principle contributor at the alveolar air-fluid interface which lowers alveolar surface tension and prevents acinar collapse on expiration (1). Without this there is alveolar collapse and as a result poor gas exchange, hypoxia, hypercarbia and acidosis. The alveolar ducts and terminal bronchioles are distended and lined by hyaline membranes which contain fibrin, cellular debris and fluid, thought to arise from a combination of ischaemia, barotrauma and the increased oxygen concentrations used in assisted ventilation (2) .

Clinically these premature infants are usually symptomatic within minutes of birth with grunting, retractions, cyanosis and tachypnoea. Chest radiographic findings may be present shortly after birth but occasionally the maximum features may not be present until 6 to 24 hours of life. Prior to the commencement of treatment, the typical radiographic features include underaeration of the lungs, fine granular opacification which is diffuse and symmetrical and air bronchograms, due to collapsed alveoli interspersed with distended bronchioles and alveolar ducts (Fig. 1a). When there is less distension the granularity is replaced by more generalized opacification or complete whiteout of the lungs (Fig. 1b). Atelectasis is the main cause of this opacification but in the very premature infant in particular, oedema, haemorrhage and occasionally superimposed pneumonia contribute.

Very premature infants, less than 26 weeks gestation may have clear lungs or mild pulmonary haziness initially. Their lungs are structurally and biochemically immature and require prolonged ventilatory support. Prenatal corticosteroid administration during the 2 days prior to delivery significantly reduces the incidence of IRDS in premature infants. The clinical use of artificial surfactant, given 

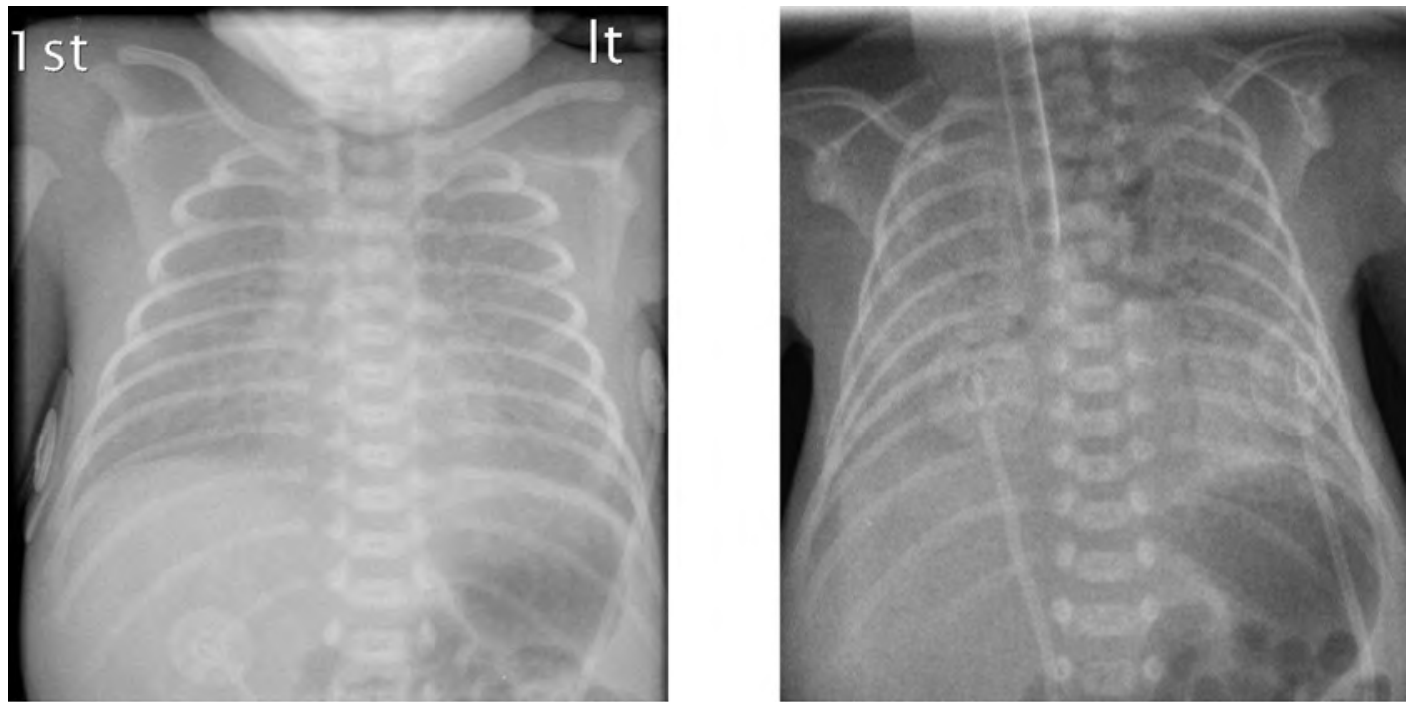

Fig. 1 (a) Infant born at 26 weeks gestation. There is moderate fine granular opacification uniformly distributed throughout both lungs with poor lung inflation. (b) Infant born at 25 weeks gestation. There is almost complete "whiteout" of both lungs with air bronchogram bilaterally.

as a liquid bolus through the endotracheal tube, has been a major therapeutic advance. It may not be evenly distributed throughout the lungs leading to areas of atelectasis interspersed with areas of good aeration (Fig. 2), and may produce radiographic findings similar to neonatal pneumonia or pulmonary interstitial emphysema (PIE). Correlation with the clinical picture in addition to the timing of the administration of the surfactant is therefore very important (Fig. 3).

In general, infants greater than 27 weeks gestation respond best to surfactant therapy. In the very premature infant, less than 27 weeks gestation, the lungs become clear following surfactant administration, but they are still immature with fewer alveoli than normal. This results in inadequate gas exchange, leads to prolonged ventilation, hazy lung opacification and occasionally a picture similar to that seen in bronchopulmonary dysplasia.

A patent ductus arteriosus is frequent in the premature infant and contributes to the disease. When IRDS is present the rigid lungs, associated hypoxia and hypercarbia

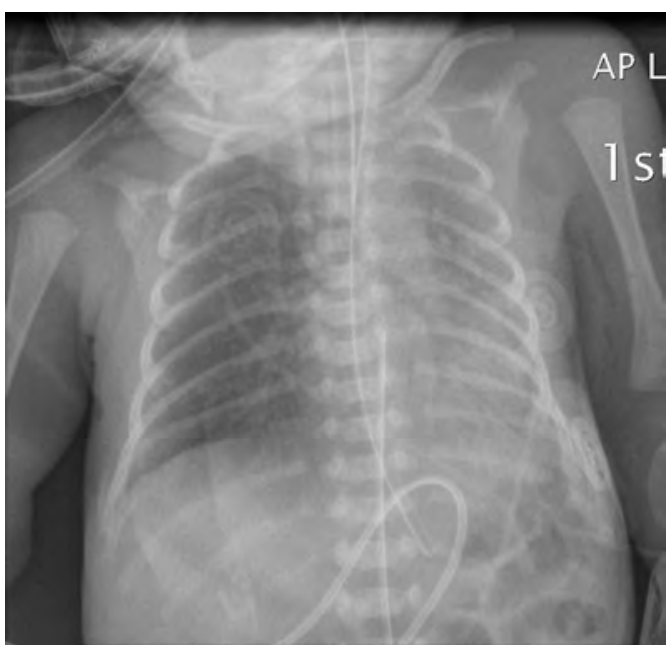

Fig. 2 Chest radiograph on a premature infant with severe HMD following administration of one dose of surfactant. There is aeration of the right lung only.

may lead to right- to-left shunting through the ductus. With surfactant therapy and improved oxygenation there is reduced pulmonary resistance and as a result there may be left-to-right shunting. Initial treatment if required is with ibuprofen, which inhibits prostaglandin production, but surgery may occasionally be required. The chest ra- 

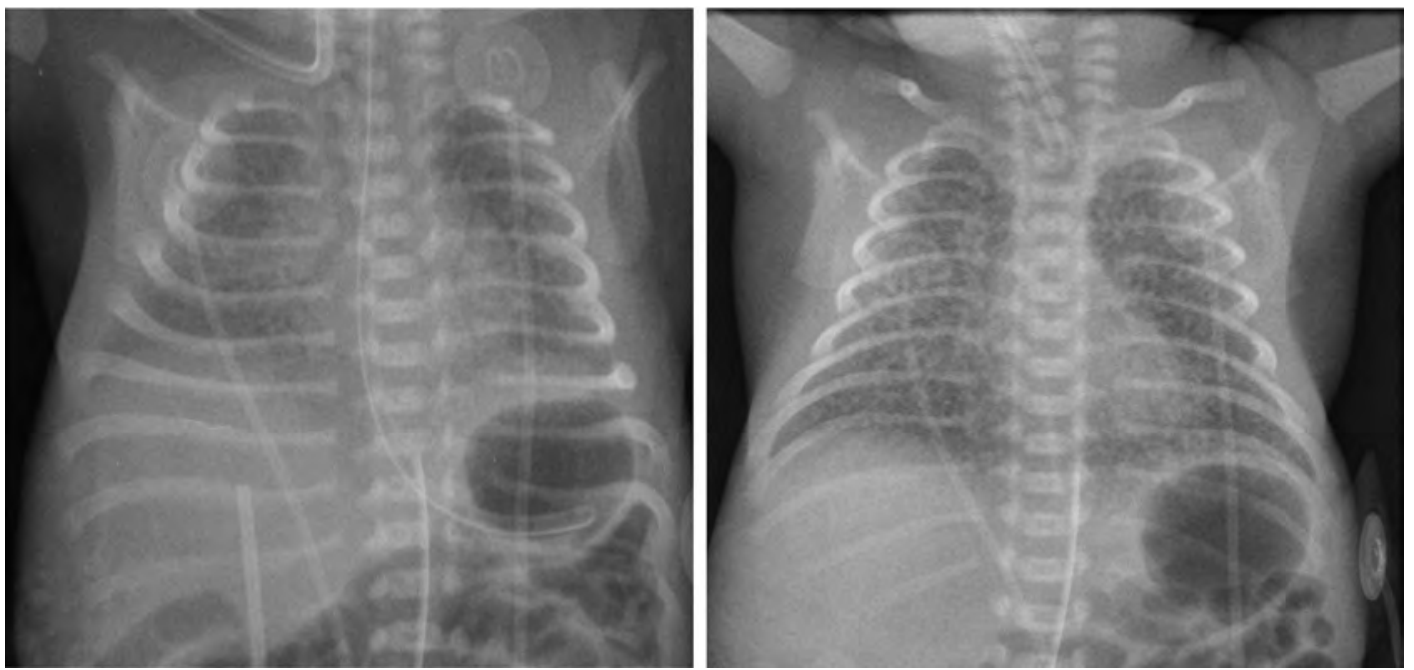

Fig. 3 (a) Premature infant with moderate HMD. (b) Radiograph performed immediately following surfactant administration demonstrates bilateral coarse pulmonary opacification as a result of the liquid surfactant in the distal air spaces.
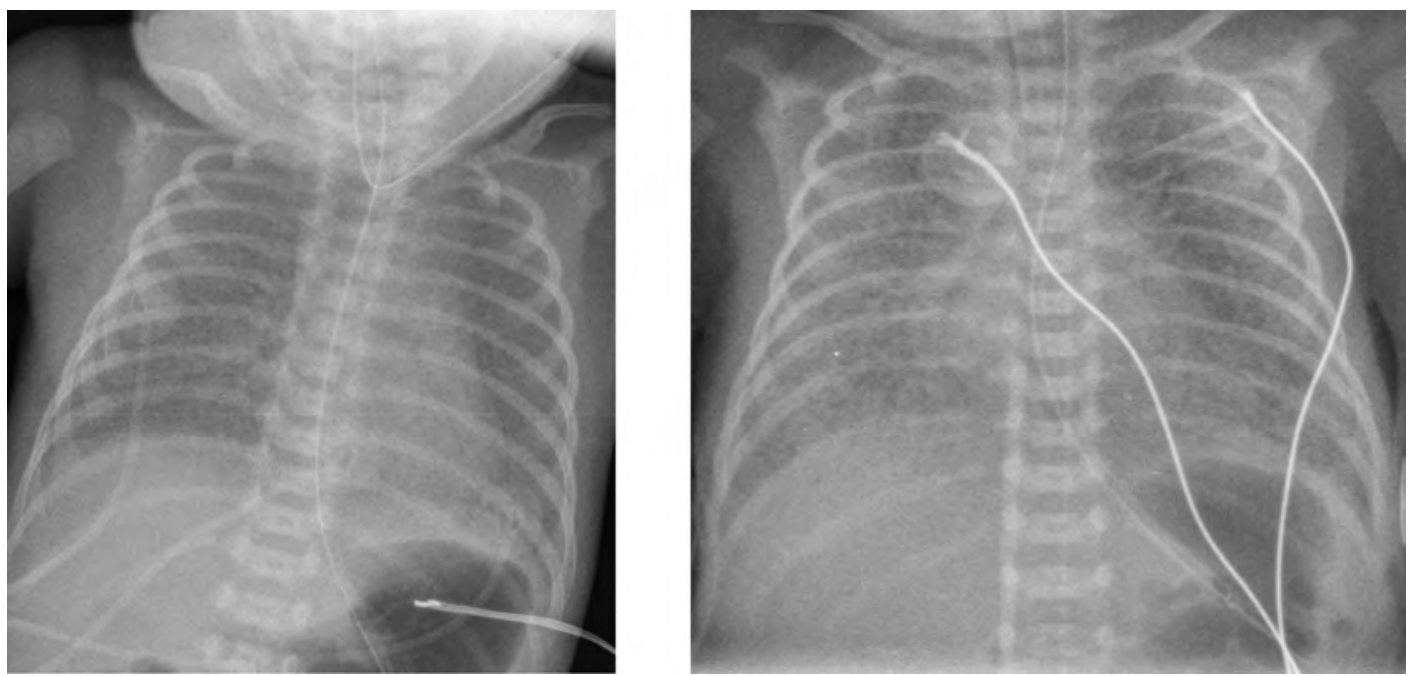

Fig. 4 (a) Premature infant with mild HMD (b) Chest radiograph at Day 3 of life demonstates a slight increase in heart size, some splaying of the carina, central blood vessel prominence and increase in lung opacification in keeping with a left to right shunt at ductus arteriosus level and pulmonary oedema.

diograph may demonstrate sudden cardiac enlargement, left atrial enlargement causing elevation of the left main bronchus and varying degrees of pulmonary oedema (Fig. 4).

There is an increasing use of prophylactic continuous positive airway pressure (CPAP) ventilation in infants suspected of developing IRDS which helps reduce the incidence of complications in these infants. High frequency ventilation is also used to reduce the incidence of barotrauma, particularly in the very premature infant. In these infants the radiographs do not differ significantly to those infants receiving conventional ventilation. The chest radiograph is used to assess the degree of lung inflation. The dome of the 


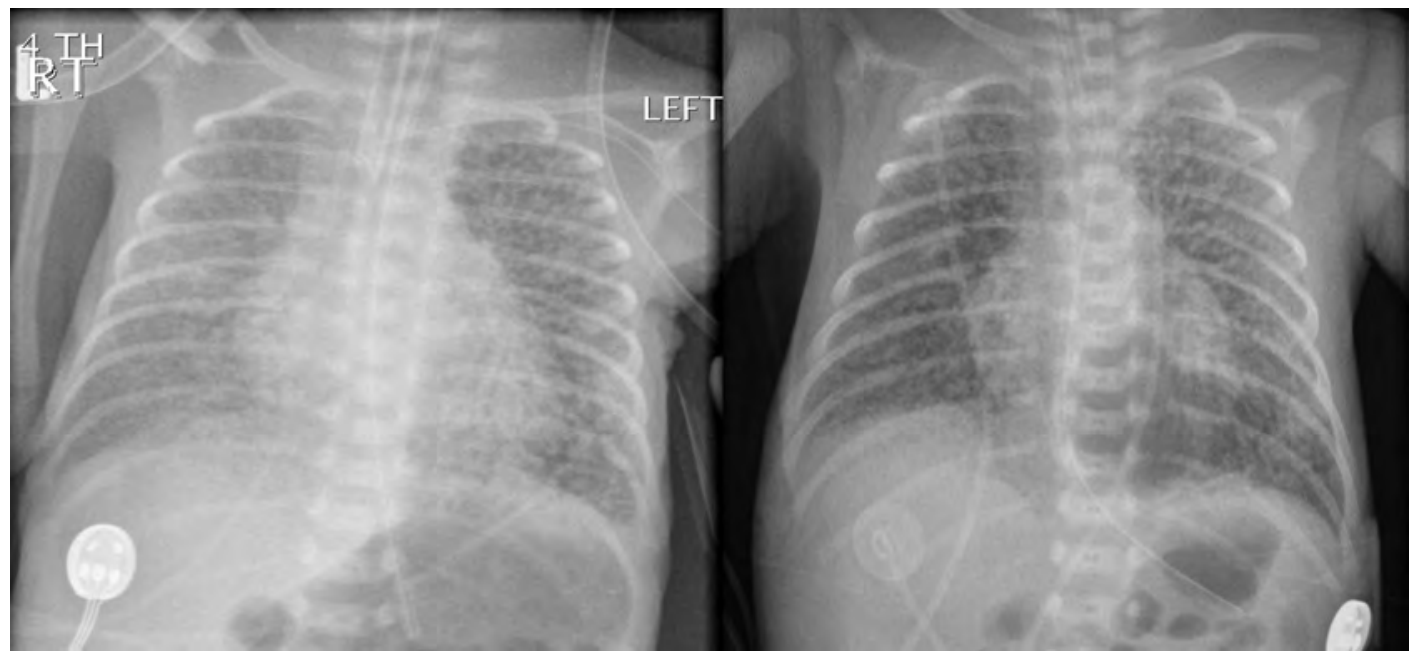

Fig. 5 (a) Premature infant with HMD. Chest radiograph at day 3 of life shows circular and linear areas of hyperlucency in the left lung due to pulmonary interstitial emphysema. (b) Another premature infant with pulmonary interstitial emphysema in both lungs. In addition, there is a large emphysematous bulla in the left lower lobe.
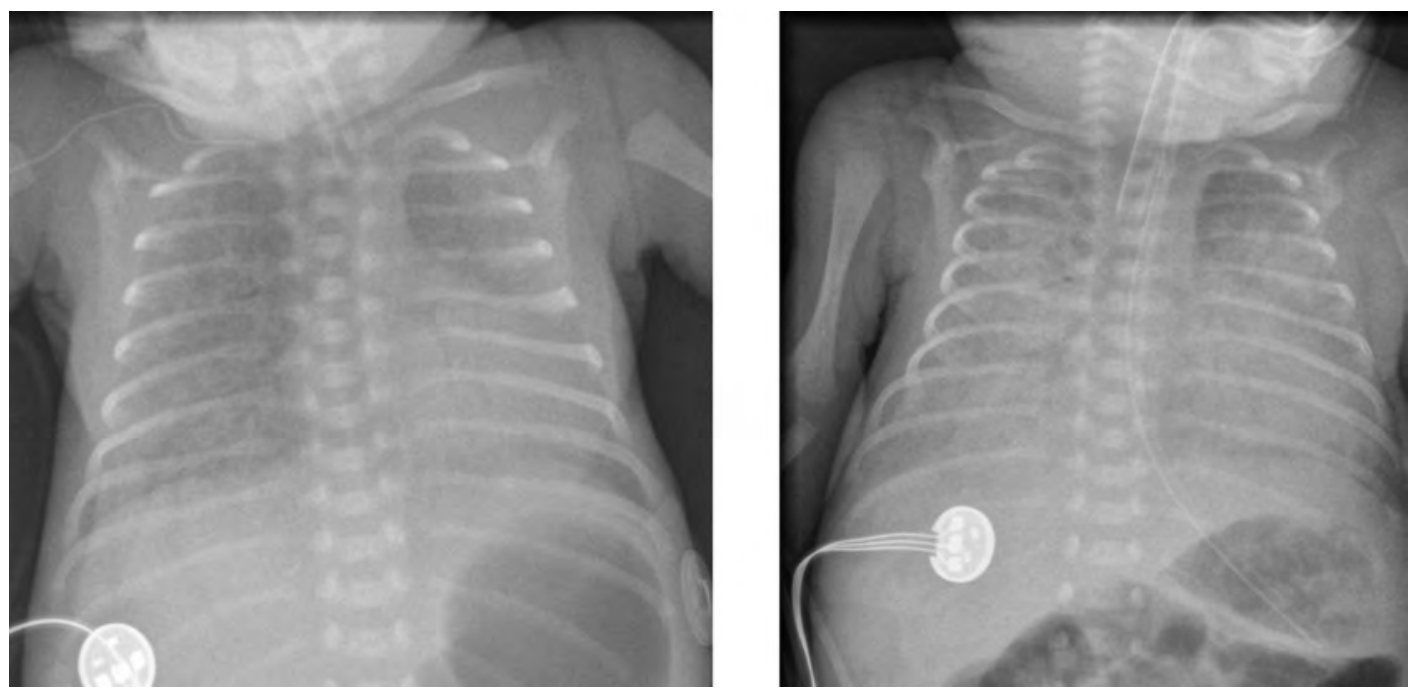

Fig. 6 (a) Premature infant with mild HMD. (b) Chest radiograph following sudden detioration at Day 4 of life with blood oozing from the ET tube. There is marked new pulmonary opacification most in keeping with pulmonary haemorrhage.

diaphragm should project at the level of the $8-10^{\text {th }}$ posterior ribs if the mean airway pressure is appropriately adjusted.

The use of positive pressure ventilation in the newborn is the most common cause of pneumothorax, pneumomediastinum, pulmonary interstitial emphysema (Fig. 5) and pneumopericardium. These complications have become much less common in infants who have been treated with surfactant and high frequency ventilation. Areas of atelectasis can occur in surfactant deficiency and is frequently due to poor clearance of secretions. Premature infants are at an increased risk of pneumonia and it may co-exist with IRDS. Pulmonary haemorrhage resulting in 
airspace opacification may also be a superimposed problem, and is usually due to severe hypoxia and capillary damage (Fig. 6).

Bronchopulmonary dysplasia (BPD) or chronic lung disease is a significant longterm complication of IRDS. Because of the many advances in neonatal care, its incidence and severity has reduced significantly in infants born at 28 weeks gestation or older. The unchanged overall incidence is due to the increased survival of the infants of extreme prematurity as they require more prolonged ventilation. Air leaks, patent ductus arteriosus and infection are contributing factors as they also prolong ventilation. Infants who develop BPD have a persisting inflammatory response and a higher incidence of BPD has been demonstrated in infants with previous culture proven Ureaplasma urealyticum pneumonitis (3). Chorioamnionitis is also considered as a risk factor. The exact definition of BPD is not clear. The four classic stages of BPD described by Northway (4) are now very rarely seen, and the definition has been modified since its initial description in 1967. More recently a consensus workshop redefined BPD to include a severity grading system which takes into account the infants gestational age (5).

Nowadays the most common radiographic appearance is diffuse interstitial shadowing with mild to moderate hyperinflation of gradual onset (Fig. 7).

A new type of BPD was described by Jobe in 1999 (6) in immature infants with minimal lung disease at birth, and who become symptomatic in the first week of life. This entity seems inseparable from the condition described previously as Wilson -Mikity syndrome. Currently, the incidence of BPD is approximately $30 \%$ in infants born at less than 1,000 grs and approximately 37\% of these have accompanying pulmonary hypertension, which is presumably linked to global hypoxia. In most patients the condition resolves over the first 12 months of life.

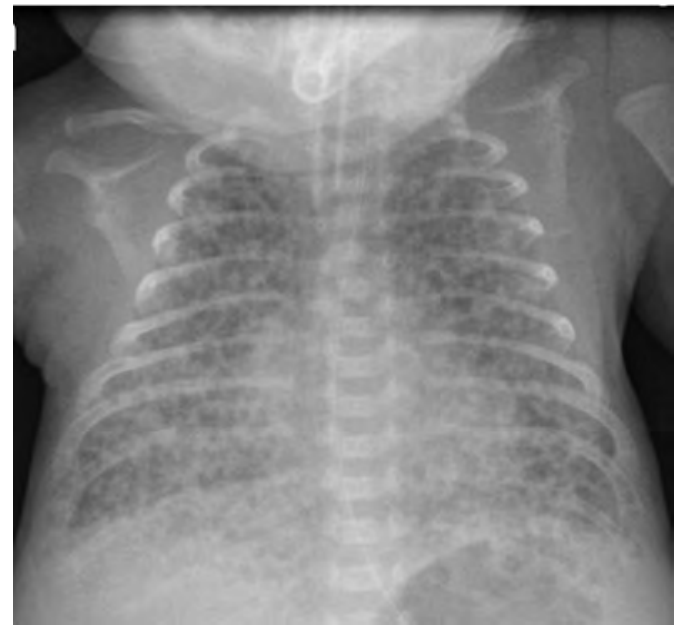

Fig. 7 Infant born at 24 weeks gestation. Chest radiograph at 8 weeks of age shows lung hyperinflation, coarse alveolar and interstitial opacification due to BPD.

Oxygen, and inhaled Nitric Oxide have been used to actively manage these patients. More recent studies have suggested that the drug Sildenafil which is a phosphodiesterase- $\mathrm{V}$ inhibitor, can be beneficial.

\section{Transient tachypnoea of the newborn (TTN)}

This condition is also referred to as retained foetal lung fluid or wet-lung syndrome. Normally fluid is cleared from the lungs at, or shortly after birth by the pulmonary lymphatics and capillaries. In TTN the normal physiological clearance is delayed. The incidence is greater in infants delivered by caesarian section, in hypoproteinaemia, hyponatraemia and maternal fluid overload. There is also an increased incidence in small, hypotonic and sedated infants who have had a precipitous delivery. Typically the infants have mild to moderate respiratory distress without cyanosis in the first couple of hours. The process resolves rapidly with almost complete resolution in 48 hours. Treatment consists of supportive oxygen and maintenance of body temperature. Radiographically, the most common findings are mild 

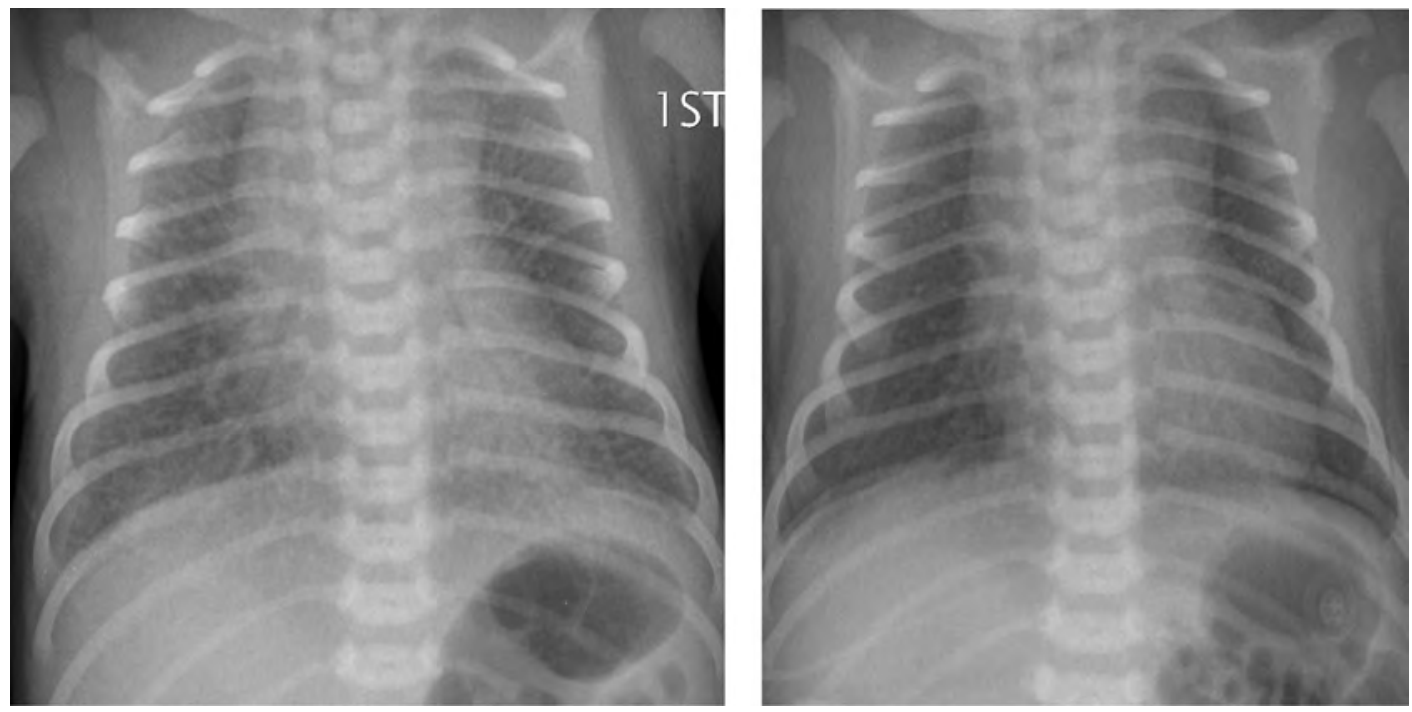

Fig. 8 Term infant with TTN. (a) Initial chest radiograph shows good lung inflation, blood vessel prominence, small bilateral pleural effusions and mild bilateral pulmonary opacification most marked at the lung bases. (b) There is almost complete resolution at 24 hours.

overinflation, prominent blood vessels, perihilar interstitial shadowing and fluid in the transverse fissure with occasional small pleural effusions (Fig. 8). There may be mild associated cardiomegaly. The appearances may be asymmetrical with right sided predominance, which remains unexplained. The features may simulate meconium aspiration syndrome and congenital neonatal pneumonia, particularly when severe.

\section{Meconium aspiration syndrome}

The definition of meconium aspiration syndrome is an infant born through meconium stained amniotic fluid where the symptoms cannot be otherwise explained (7). It is thought that fetal hypoxia causes foetal intestinal hyperperistalsis and passage of meconium which is aspirated by a gasping foetus. It is most common in infants who are postmature. It is diagnosed by the presence of meconium below the level of the vocal cords. The chest radiographic features may in part be due to the inhalation of meconium itself in utero or during birth. It is a thick viscous substance and may lead to areas of atelectsais and overinflation. It may migrate to the distal airways causing complete or partial obstruction and lead to a "ball-valve" effect. It may also cause a chemical pneumonitis (Fig. 9). There may be associated alterations in the pulmonary vasculature leading to pulmonary arterial hypertension. Air leaks are common and small associated pleural effusions may be seen. Many of the infants will require mechanical ventilation. The mortality rate has been improved by the use of inhaled nitric oxide, to treat severe pulmonary hypertension and also by Extracorporeal membrane oxygenation (ECMO), which is used only in those infants where the conventional treatments have failed. The ECMO technique can be either with veno-arterial method where one catheter is placed in the internal jugular vein and one in the carotid artery (Fig. 10) or veno-venous method where a double lumen catheter is placed in the internal jugular vein, superior vena cava or right atrium. The circulation bypasses the lungs which are minimally inflated and allows physiologic levels of oxygen saturation. 


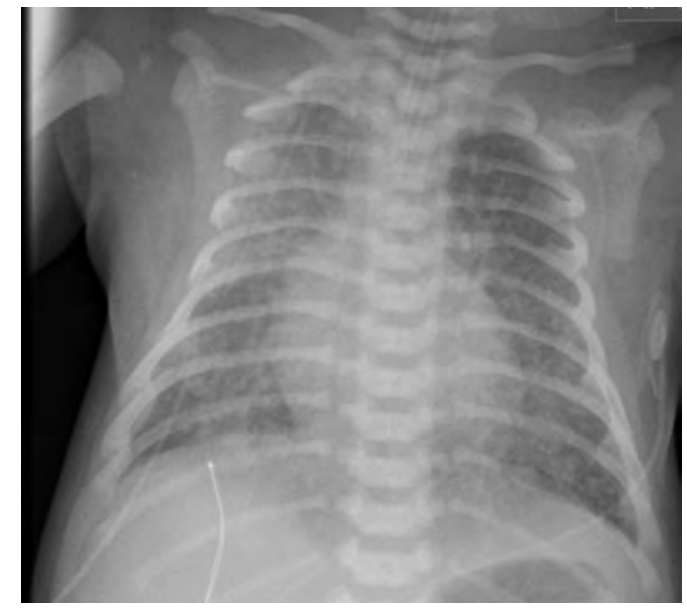

Fig. 9 Chest radiograph on a term infant with meconium aspiration syndrome. There is marked coarse opacification in the lungs, most marked in the right upper lobe.

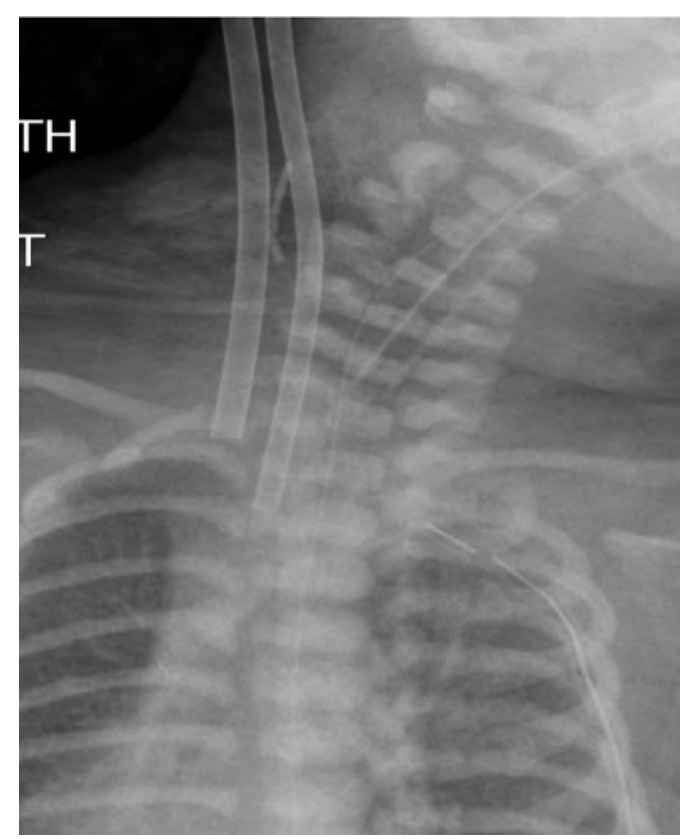

Fig. 10 Radiograph demonstrating correct positioning of veno-arterial catheters in an infant undergoing ECMO.

\section{Congenital neonatal pneumonia}

Neonatal infections acquired transplacentally, such as TORCH (toxoplasmosis, rubella, cytomegalovirus, herpes) are rare and seldom develop pulmonary abnormalities. Infections acquired perinatally can occur via ascending infection from the vagina, transvaginally during birth or as a hospital acquired infection in the neonatal period. Prolonged rupture of membranes prior to delivery is a major risk factor. It is thought that most cases of neonatal pneumonia occur during birth, when the infant may swallow and/or aspirate infected amniotic fluid or vaginal tract secretions. Group B streptococcus is the most common organism identified. The radiological features are non-specific. The most common features seen on the chest radiograph in term infants who present with severe acute symptoms in the first 24 to 48 hours are coarse bilateral asymmetrical alveolar opacification (Fig. 11) with or without associated interstitial change. In these infants the radiographic changes may mimic meconium aspiration syndrome or severe transient tachypnoea. The presence of pleural effusions, pulmonary hyperinflation and mild cardiomegaly may not be helpful in differentiating pneumonia from these other conditions.

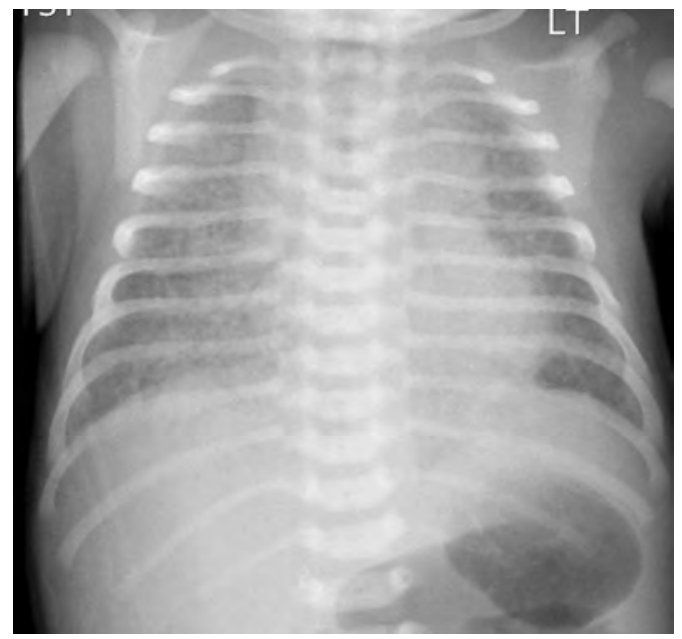

Fig. 11 Infant with congenital group B streptococcus pneumonia. There is asymmetrical bilateral coarse pulmonary opacification, most marked in the right middle lobe.

In the premature infant there maybe diffuse fine granular opacification, similar to the 
appearances seen in idiopathic respiratory distress syndrome (IRDS) (8). Some infants may have both IRDS and group B streptococcus pneumonia. Clamydial infection classically presents first with conjunctivitis at 1-2 weeks after birth and the lung infection does not usually become evident until 4 to 12 weeks of age. Typically the radiograph demonstrates interstitial opacification with some hyperinflation. The association of ureaplasma urealyticum with neonatal pneumonia is increasingly recognized. These infants have a mild early course and develop features of BPD at an earlier age than would be expected in a premature infant (9).

\section{Spontaneous air leaks}

Spontaneous pnuemothoraces and pneumomediastinum may cause respiratory distress in the newborn infant. Many are transient and do not require intervention. A pneumothorax may be radiographically subtle in sick infants as supine radiographs are usually performed and free air accumulates over the lung surface producing a hyperlucent lung and increased sharpness of the mediastinum. A pneumomediastinum usually outlines the thymus (Fig. 12) and when there is a pneumopericardium the air surrounds the heart.

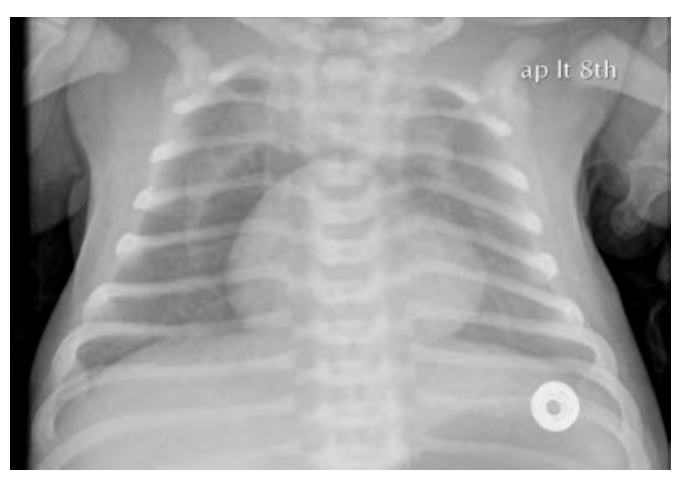

Fig. 12 Infant with a spontaneous pneumomediastinum. The inferior boarder of the thymus is clearly outlined and is elevated.

\section{Persistent pulmonary hypertension of the newborn (PPHN)}

PPHN is the result of failure of the normal pulmonary vascular relaxation which occurs at or shortly after birth. There is high pulmonary vascular resistance, right-to-left shunting and severe hypoxaemia. It may occur in isolation such as when associated with perinatal stress and may occur in association with parenchymal abnormalities, such as meconium aspiration, pneumonias and congenital lung abnormalities. There are no specific chest radiographic findings. The radiograph often shows the underlying cause, but the lungs may look clear or have mild perihilar hazy opacification. The diagnosis is confirmed by performing echocardiography

\section{Surfactant deficient disorders}

Disorders of surfactant deficiency due to a genetic abnormality in the surfactant protein $\mathrm{B}(\mathrm{SpB})(10)$ and $\mathrm{C}(\mathrm{SpC})(11)$ and the ATP-binding cassette transporter protein A3 (ABCA3) can lead to interstitial lung disease. Inherited mutations in the $\mathrm{SpB}$ and $\mathrm{ABCA} 3$ are autosomal recessive and may present immediately after birth with respiratory symptoms. Mutations in the $\mathrm{SpC}$ are autosomal dominant and may present later in infancy. The chest radiograph may show diffuse hazy opacification initially with the later development of interstitial shadowing which may be progressive (Fig. 13a). CT demonstrates diffuse ground-glass opacification with septal thickening (12) and cystic change (Fig. 13b). Pulmonary interstitial glycogenosis (PIG) may present in the preterm or term infant very soon after birth. It has been reported in isolation but is frequently associated with conditions that affect lung growth and the diagnosis is made by the pathological examination of lung tissue. The imaging features may be similar to those seen in the other disorders of surfactant deficiency. 

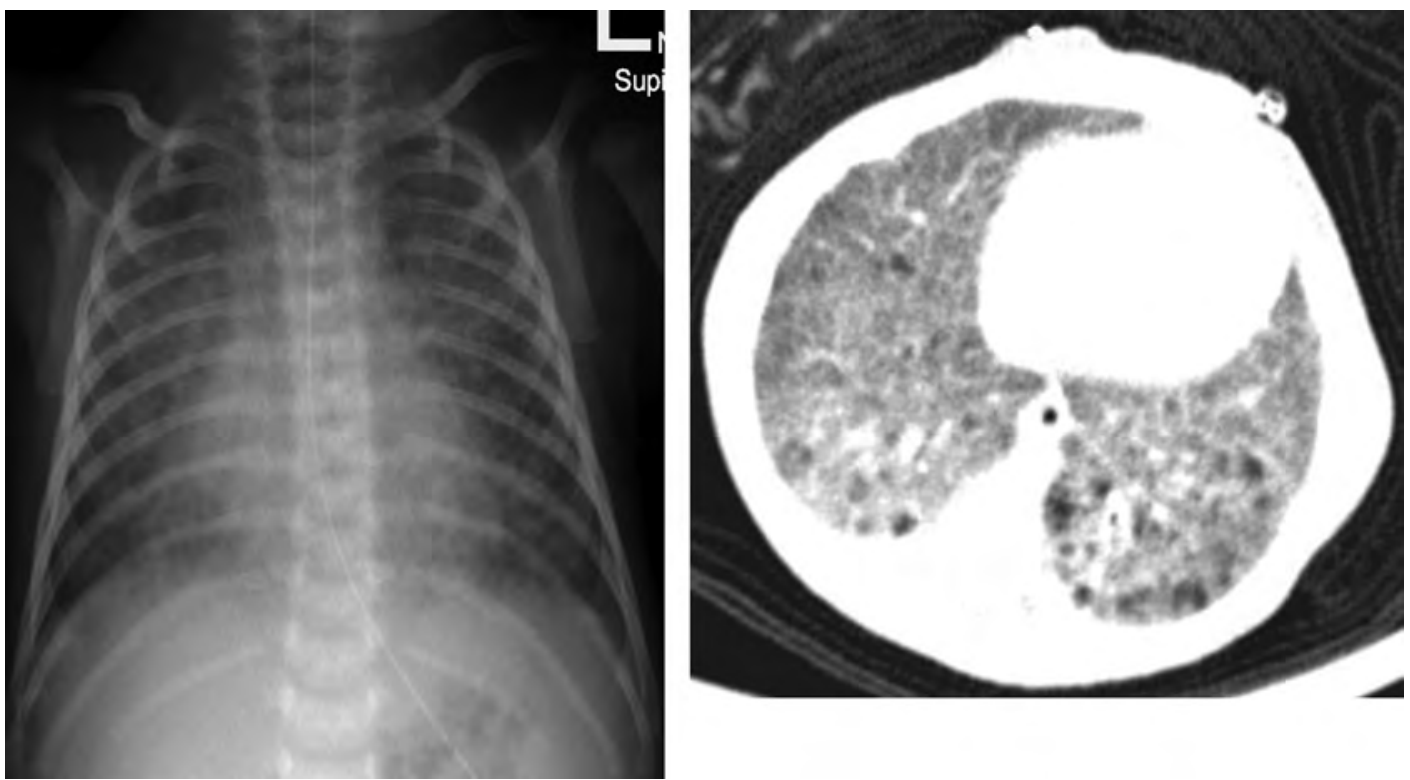

Fig. 13 Infant with $A B C A 3$ surfactant disorder. Chest radiograph at 1 month of age shows bilateral alveolar and interstitial opacification. (b) CT shows diffuse ground-glass opacification with some cystic change.

\section{Pulmonary hypoplasia}

Pulmonary hypoplasia is due to the underdevelopment of the alveoli and bronchi and is commonly associated with pulmonary vessel abnormalities. If severe, it may cause severe respiratory distress at birth. In the newborn it may occur secondary to congenital diaphragmatic hernia. Other causes include congenital renal anomalies such as autosomal recessive polycystic kidney disease, skeletal dysplasia and neuromuscular disorders.

\section{Congenital abnormalities which cause mass effect and respiratory distress in the neonate}

The common congenital abnormalities in this catergory include congenital diaphragmatic hernia, congenital pulmonary airway malformations (CPAM) and congenital lobar emphysema. The vast majority of these conditions are diagnosed antenatally, and many of require surgical intervention.
In infants with CPAM the condition may shrink with time and in some cases resolve completely

\section{Congenital diaphragmatic hernia}

Congenital diaphragmatic hernia is the result of delayed closure of the pleuro-peritoneal canal in the prenatal period resulting in a delay in the return of the bowel loops to the abdominal cavity. The diagnosis is usually made antenatally using ultrasound, and occurs on the left side in approximately $90 \%$ of cases. Occasionally, MRI maybe required to help in difficult cases, particularly when the abnormality is on the right side. Postnatal radiographs initially show opacification with poor aeration of the ipsilateral lung and contralateral mediastinal displacement. With time, the characteristic air filled bowel loops fill the thorax (Fig. 14). The abdomen may have a scaphoid appearance due to the paucity of the bowel loops in the abdominal cavity. Right sided hernias may contain liver or kidney in addition to bowel loops. The progno- 
sis in these infants depends on the degree of pulmonary hypoplasia. Following the hernia repair, a chest radiograph will demonstrate the volume of the underlying lung.

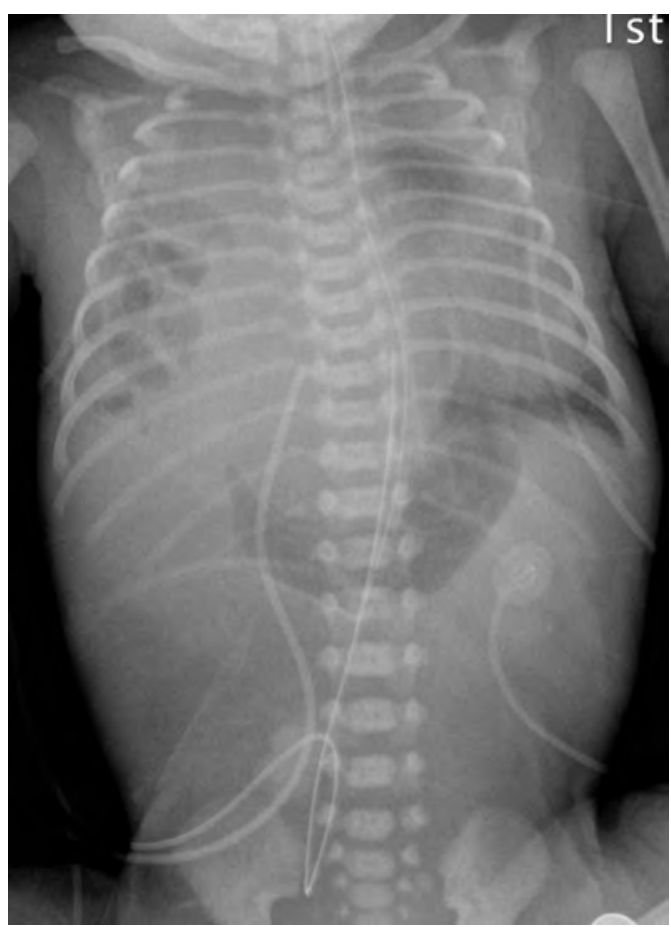

Fig. 14 Infant with right congenital diaphragmatic hernia. Chest radiograph shortly after birth,shows that the right hemidiaphragm is not visualised, and multiple air filled bowel loops occupy the right lateral hemithorax resulting in mediastinal displacement to the left. The position of the umbilical venous catheter is also elevated, an indication that the liver is partly in the right chest cavity also. There is a paucity of bowel loops in the abdominal cavity.

\section{Congenital eventration of the diaphragm}

A localised weakness of part of one hemidiaphragm may bulge upwards and cause compression of the adjacent lung. It may occasionally cause respiratory distress and surgical repair may be necessary.

\section{Congenital pulmonary airway malformation}

This condition follows from hamartomatous development of the terminal bronchioles. In the past the condition has been termed congenital cystic adenomatoid malformation of the lung (CCAM). It was initially classified into 3 types by Stocker et al. in 1977 but has recently been reclassified by Stocker (13) into 5 types $(0-\mathrm{IV})$, based on the location or stage of development of the lesion. Type 0 is the rarest and is due to an abnormality of the trachea and main bronchi. Type I is the commonest and is due to an abnormality of the bronchial +/- bronchiolar region. Type II involves the bronchiolar region and Type III the terminal alveolar alveolar duct region. Type IV involves the distal acinus or saccular /alveolus region. The abnormality is increasingly diagnosed in utero using ultrasound and reduction in the size of the mass is detected in the $3^{\text {rd }}$ trimester in up to $75 \%$ of cases. Many patients are asymptomatic at birth, but if the lesion is large the infant will have respiratory distress. When performed early after birth, the chest radiograph will show a dense mass causing mass effect, and may be difficult to distinguish from a congenital diaphragmatic hernia or lobar emphysema. With time the appearance is that of air filled cysts. A CT chest is very helpful in characterising the lesion and in assessing its extent (Fig. 15). In the large number of patients where the mass is shown to resolve on antenatal ultrasound and where a postnatal chest radiograph may be normal a CT scan of the chest will often demonstrate a residual lesion. If the lesion is large and causing symptoms, early surgical resection is the treatment of choice. Smaller lesions are also frequently resected due to the increased risk of superimposed infections or potential development of superimposed neoplasm such as pleuropulmonary blastoma. 

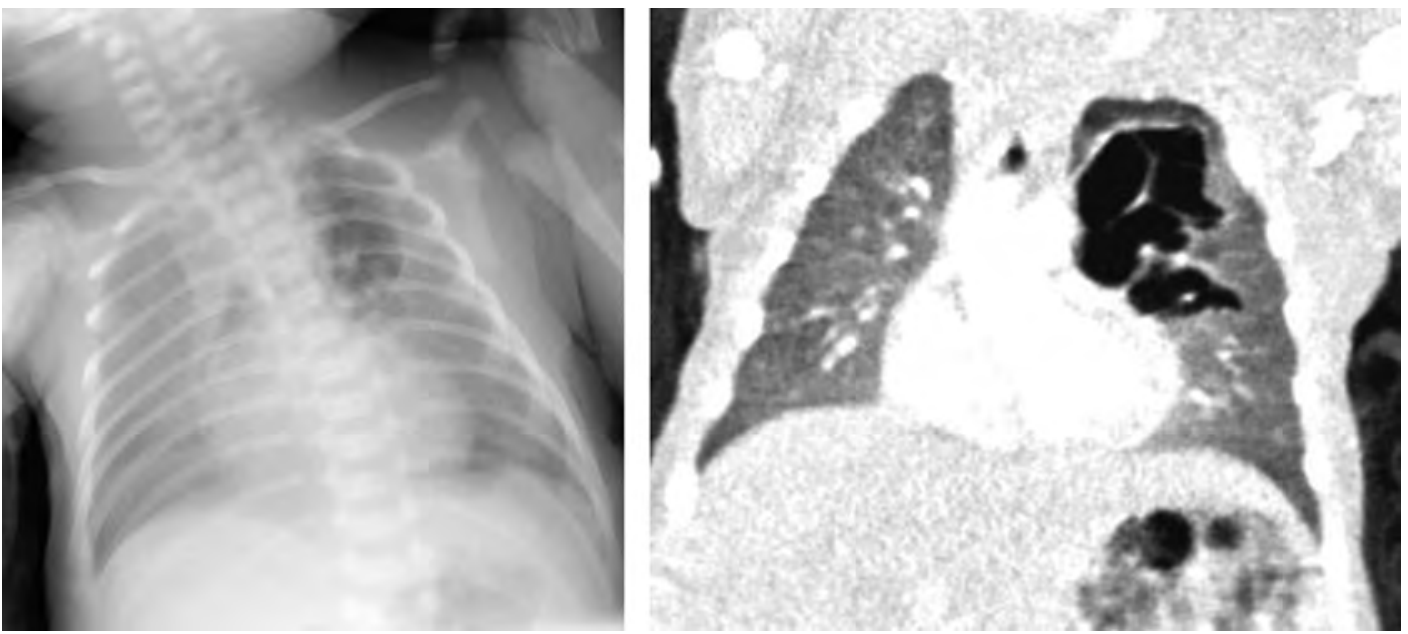

Fig. 15 Infant with antenatal diagnosis of left CPAM. Chest radiograph shortly after birth shows a multicystic mass occupying the left upper lobe. Coronal reconstruction CT image (b) outlines the extent of the abnormality.

\section{Pulmonary sequestration}

Pulmonary sequestration is an aberrant lung mass which is supplied by the systemic arteries and the venous drainage may be to the systemic or pulmonary veins or both. Most are not connected to the bronchial tree and may be intralobar oe extralobar. Extralobar sequestration is much less common than the intralobar form but is most likely to present in the newborn period. The majority are found in the left lower lobe close to the dia- phragm but they can occur on the right side and can also be located below the diaphragm, close to the adrenal gland and may mimic a neuroblastoma. Extralobar sequestrations are frequently diagnosed on antenatal ultrasonography but the arterial vessel supply is not always seen. Antenatal MRI may be helpful but this also does not always demonstrate the vessel supply. Postnatally, the Chest radiograph will show focal lung opacification. Multidetector CT following intravenous contrast administration is the technique of
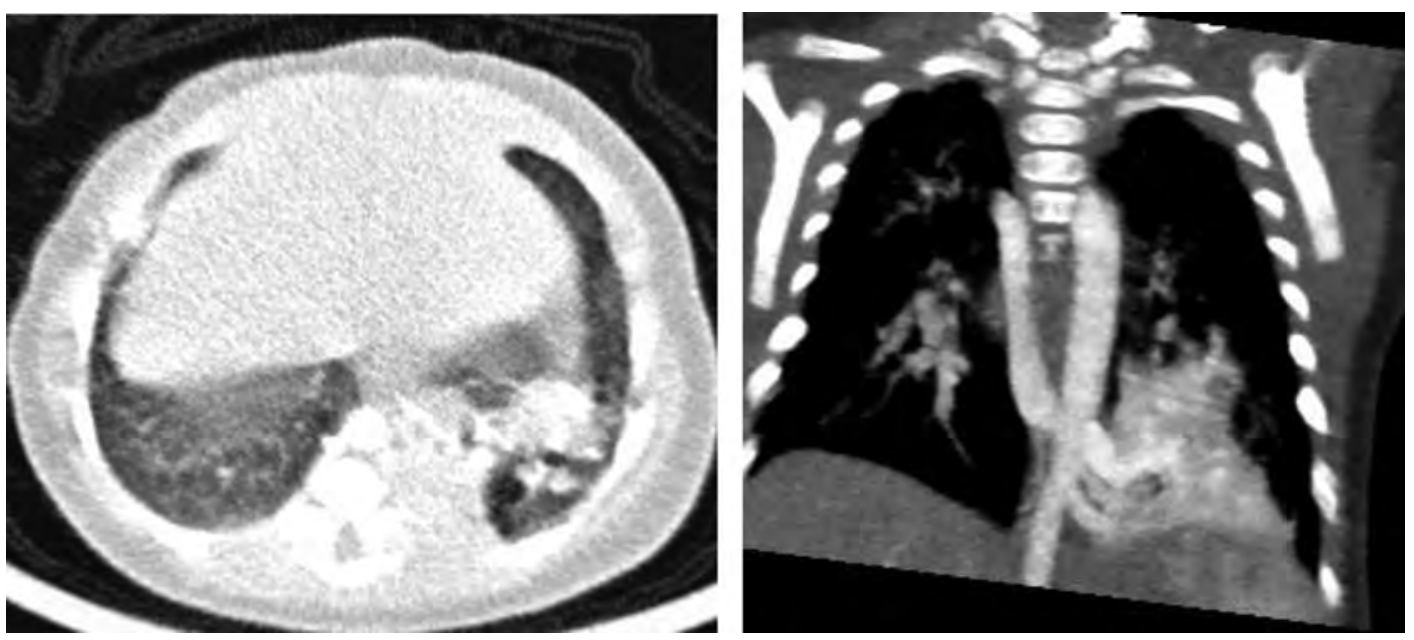

Fig. 16 (a) Axial CT chest image with a mixed solid and cystic left lower lobe mass. (b) Coronal reconstruction image shows the left lower lobe mass with a feeding vessel arising from the aorta. 
choice to confirm the diagnosis (Fig. 16) as the spatial resolution of small vessels is better than on MRI and the examination can most often be performed without sedation or anaesthesia. Surgical resection is the current treatment of choice in children with pulmonary sequestrations.

\section{Hybrid lesions}

Hybrid lesions are made of extralobar pulmonary sequestrations and CPAM Type I and are common (14) (Fig. 16). They can also be located below the diaphragm.

\section{Congenital lobar overinflation}

The condition is also known as congenital lobar emphysema. The aetiology is uncertain but is presumably due to an abnormality of a lobar bronchus which may have an obstructing cartilage abnormality (15). It most commonly affects the left upper lobe, followed by the middle lobe and the right upper lobe. There is usually progressive hyperinflation

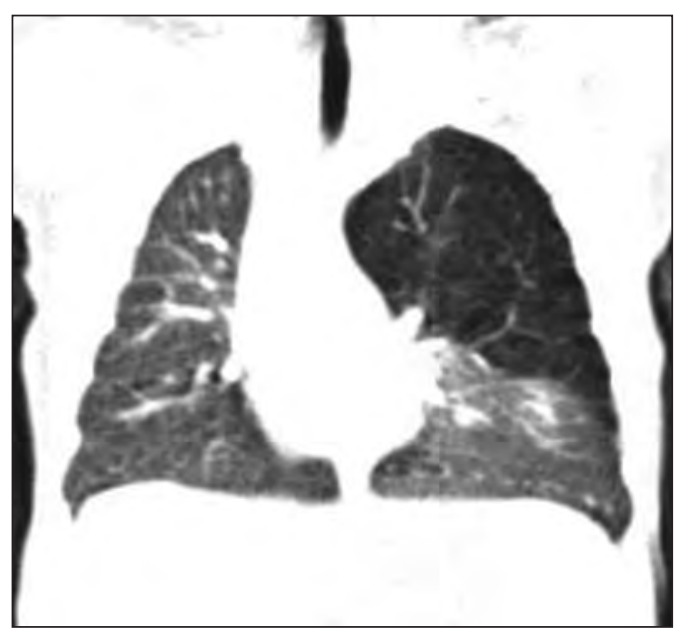

Fig. 17 Coronal reconstruction CT images in a 5 week old infant with dyspnoea at rest, showing hyperinflation of the left upper lobe causing mediastinal displacement to the right. Following left upper lobe resection a diagnosis of congenital lobar overinflation was made. of the affected lobe leading to progressive mass effect on the adjacent structures. Most patients present during the neonatal period with respiratory distress. There are associated congenital abnormalities, such as cardiovascular abnormalities in up to $14 \%$ of patients. Early in the newborn period the chest radiograph may demonstrate opacification due to retained lung fluid. Typically, there is hyperlucency with varying degrees of displacement of adjacent lobes and mediastinal structures (Fig. 17). CT of the chest is helpful to confirm the abnormality and confirm the exact lobe involved. Surgical resection is the treatment of choice in symptomatic patients.

\section{Pleural effusions}

The most common cause of a congenital pleural effusion is chylothorax. The cause is unknown, and late maturation of the thoracic duct has been suggested as an aetiology. The abnormality is usually detected on antenatal ultrasonography and in utero drainage may be performed to prevent pulmonary

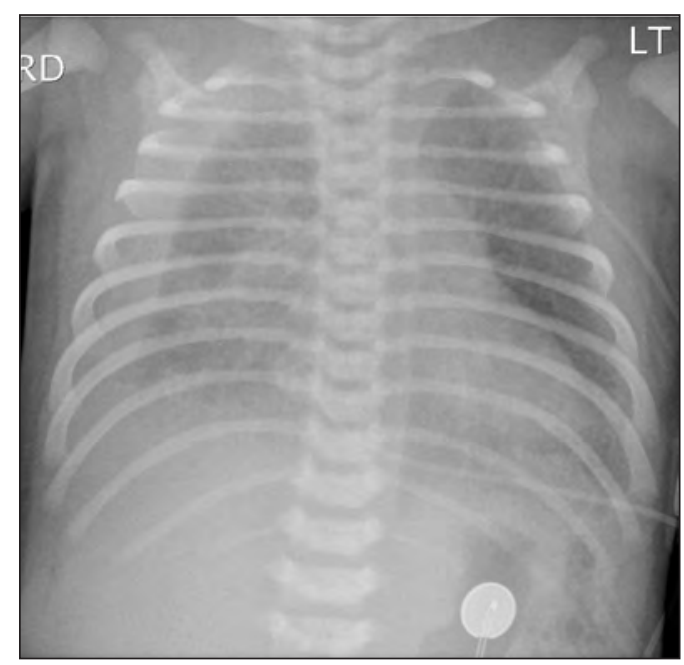

Fig. 18 Postnatal chest radiograph in a newborn with an antenatal diagnosis of bilateral pleural effusions, showing a small left and a large right pleural effusion, tracking along the lateral chest wall. Aspiration confirmed chylothoraces. 
hypoplasia. Postnatally, the chest radiograph demonstrates the pleural effusions (Fig. 18). Aspirated fluid will have a high lymphocyte count but will not have a milky appearance until such time as the infant is fed with fat. Resolution is usually complete but often after multiple aspirations.

\section{Conclusion}

Lung abnormalities are not uncommon in the premature and term infants and there have been many improvements in the management of these often very ill newborns. Chest imaging, in particular chest radiography plays a pivotal role in the diagnosis of these conditions and in the monitoring of complications in these infants. Advances in fetal ultrasonography and MRI lead to more frequent diagnoses of congenital lesions which affect the lungs and have led to a better understanding of their evolution. This allows better planning of postnatal imaging and postnatal clinical and surgical care.

Conflict of interest: The author declares that she has no conflict of interest.

\section{References}

1. Agrons GA, Courtney SE, Stocker JT, Markowitz RI. From the archives of the AFIP: Lung disease in premature neonates: radiologic-pathologic correlation. Radiographics. 2005;25(4):1047-73.

2. Wood BP, Davitt MA, Metlay LA. Lung disease in the very immature neonate: radiographic and microscopic correlation. Pediatr Radiol. 1989;20:33-40.

3. Wang EEL, Matlow AG, Ohlsson A.Ureaplasma urealyticum infections in the perinatal period. Clin Perinatol. 199724:91-105.

4. Northway WH Jr, Rosan RC, Porter D. Pulmonary disease following respirator therapy of hyaline membrane disease: bronchopulmonary dysplasia. N Engl J Med. 1967;276:357-68.

5. Jobe AH, Bancalari E. Bronchopulmonary Dysplasia. Am J Resp Crit Care Med. 2001;163:1723-29.

6. Jobe AH. The new BPD: an arrest of lung development. Pediatr Res. 1999;66:641-3.
7. Cleary GM, Wiswell TE. Meconium - stained amniotic fluid and the meconium aspiration syndrome. An update (review). Pediatr Clin North Am. 1999;45:511-29.

8. Ablow RC, Driscoll SG, Effmann EL, Gross I, Jolles CJ, Uauy R, et al. A comparison of earlyonset group B steptococcal neonatal infection and the respiratory-distress syndrome of the newborn. N Engl J Med. 1976;294(2):65-70.

9. Theilen U, Lyon AJ, Fitzgerald T, Hendry GM, Keeling JW. Infection with Ureaplasma urealyticum: is there a specific clinical and radiological course in the preterm infant? Arch Dis Child Fetal Neonatal Ed. 2004;89(2):F163-7.

10. Cole FS, Hamvas A, Rubinstein P, King E, Trusgnich M, Nogee LM, deMello DE, Colten HR. Population-based estimates of surfactant protein B deficiency. Pediatrics. 2000;105(3 Pt 1):538-41.

11. Garmany TH, Wambach JA, Heins HB, WatkinsTorry JM, Wegner DJ, Bennet K, et al. Population and disease-based prevalence of the common mutations associated with surfactant deficiency. Pediatr Res. 200;63(6):645-9.

12. Doan ML, Guillerman RP, Dishop MK, Nogee LM, Langston C, Mallory GB, Sockrider MM, Fan LL. Clinical, radiological and pathological features of ABCA3 mutations in children. Thorax. 2008;63(4):366-73.

13. Stocker J. Congenital Pulmonary Airway Malformation: a new name and expanded classification of congenital cystic adenomatoid malformations of the lung. Histopathology; 2001;41(Suppl 2):424-58.

14. Conran RM, Stocker JT. Extralobar sequestration with frequently associated congenital cystic adenomatoid malformation, type 2: Report of 50 cases. Pediatr Develop Pathol. 1999;2:454-63.

15. Berrocal T, Madrid C, Novo S, Gutiérrez J, Arjonilla A, Gómez-León N. Congenital anomalies of the tracheobronchial tree, lung, and mediastinum: embryology, radiology, and pathology. Radiographics. 2004;24(1):e17.

\section{Suggested further reading}

1. Radiological Imaging of the Neonatal Chest V. Donoghue Ed. 2nd revised edition (2008) Springer-Verlag Berlin Heidelberg New York

2. Imaging in Pediatric Pulmonology Robert $\mathrm{H}$ Cleveland Ed 2012 Springer - Verlag New York Dordrecht Heidelberg London. 